\title{
Opinion
}

\section{Successful rehabilitation in patients with postoperative maxillofacial defects}

\author{
Ubaydullaev K Asatullaevich $^{1)}$, Hiromichi Maeda ${ }^{2)}$, Rizaev J Alimdjanovich ${ }^{3)}$, \\ Gafforov S Amrulloevich ${ }^{4)}$, Gafur-Akhunov M-A Aliyarovich ${ }^{5)}$, Junichi Sakamoto ${ }^{6)}$ \\ ${ }^{1)}$ Republican Oncological Research Center, Tashkent Institute of Postgraduate Medical Education, Tashkent, Uzbekistan. \\ ${ }^{2)}$ Department of Surgery, Kochi Medical School Hospital, Nankoku, Japan. \\ ${ }^{3)}$ Tashkent State Dental Institute, Tashkent, Uzbekistan. \\ ${ }^{4)}$ Stomatology, pediatric dentistry and orthodontics department, Tashkent Institute of Postgraduate Medical Education, \\ Tashkent, Uzbekistan \\ ${ }^{5)}$ Oncology Department, Tashkent Institute of Postgraduate Medical Education, Republican Oncological Research Center, \\ Tashkent, Uzbekistan. \\ ${ }^{6)}$ Tokai Central Hospital, Kakamigahara, Japan
}

\begin{abstract}
Purpose: Previously, we demonstrated the efficacy of rehabilitation programs after maxillofacial surgery. In the present study, we extracted and summarized recommendations for the successful and comprehensive treatment of malignant tumors in the maxillofacial area.

Material and methods: We analyzed the medical histories and ambulatory cards of 107 oncological patients with postoperative maxillofacial defects who underwent various prosthetic procedures.

Results: Using polyurethane plastics to produce a protective plate on the day of surgery is necessary to improve the shortterm results of orthopedic treatment. The classification of the defect, selection of prosthesis according to the classification, applying the prosthesis without delay, and functional restoration by articulation gymnastics and mechanotherapy compose of the comprehensive approach for the defect at maxillofacial area after surgery.

Conclusions: We propose that integrating the benefits from each treatment method and rehabilitation process would further improve quality of life after resection of maxillofacial tumors.
\end{abstract}

Keywords: rehabilitation, MFA, defect

(Received September 9, 2019; Accepted Octorber 7, 2019)

\section{Introduction}

Postoperative defects of the maxillofacial area can cause functional deterioration of mastication, swallowing, speech, and hearing, as well as considerably distorting the patient's face and thus potentially influencing the patient's mental state ${ }^{1,2)}$. In addition, the importance of rehabilitation for these patients is increasing due to improved life expectancy with more complex and radical treatments.

Prostheses are widely used to compensate for defects in the maxillofacial area, with clinical surveys and our previous study demonstrating the three-staged prosthetic technique as a rational and efficient approach $^{3-6)}$. In addition, complex articulation gymnastics and mechanotherapy to prevent maxillary trismus support the restoration

Corresponding author: Hiromichi Maeda, MD, PhD. Department of Surgery, Koch Medical School Hospital, Kochi University, Kohasu, Oko-cho, Nankoku, Kochi, 783 8505, Japan. E-mail; hmaeda@kochi-u.ac.jp, TEL: +81-88-880-2370, FAX: +8188-880-2371. of damaged functions such as chewing, swallowing, and respiration. Such approaches are followed in our institute, and this study analyzes and presents recommendations to maximize the prosthesis treatment success.

\section{Method}

We retrospectively reviewed the medical histories and ambulatory cards of 107 oncological patients with postoperative defects of the maxillofacial area that underwent treatment in the Republican Oncology Research Center (RORC) and Tashkent Institute of Postgraduate Medical Education.

\section{Results}

\section{Patients}

The study enrolled men and women with cancer, aged 20-70 years. Most patients had stage III or IV disease, while the remaining patients were diagnosed as stage I or II. Patients were divided into three groups to prescribe 
defect-specific rehabilitation programs, as demonstrated previously ${ }^{6}$ : patients with defects of the upper jaw and intact teeth on the remaining jaw $(n=68)$; patients with defects of the upper and lower jaws with partial defects of the teeth on the remaining upper and lower jaws (n = 53); patients with extensive defects of the upper jaw, facial soft tissues, and full secondary adentia of alveolar process on the remaining upper jaw $(n=13)$.

\section{Indication for the complex orthopedic apparatus}

The following factors were carefully assessed before treatment: the possibilities of plastic surgery, accompanying disease, presence of scarred, changeable tissue around the postoperative defect, and wound infection. The patient also had to approved further surgical intervention.

\section{Objectives of primary stage of orthopedic interven- tion}

The primary treatment must ensure the return of independent eating and speech functions immediately after surgery, and restore the oral cavity functions, especially in patients with defects of both upper and lower jaws. Separation of the wound surface and oral cavity is inevitable for wound healing, and polyurethane plastics are useful at this stage for producing a protective plate on the day of surgery to improve the results of immediate orthopedic treatment. Applying antiseptic-soaked tampons the postoperative cavity should be considered.

\section{Objectives of the second stage of orthopedic interven- tion}

The second stage intervention aimed to create an adequate bed for an obturator-part of the permanent prosthesis, with success ensuring the improvement of lost functions.

\section{Objectives of the third stage of orthopedic interven- tion}

A permanent prosthesis was designed to restore oral cavity functions (chewing, swallowing, and speech), as well as the original external image of the face.

\section{Discussion}

Rehabilitation after surgical resection of malignant tumors in the maxillofacial area has increased in prominence because of improved patient life expectancies, resulting from the multidisciplinary treatment approach including radiation therapy, chemotherapy, and hypothermic treatment ${ }^{1,3,7-9)}$. Furthermore, therapeutic psychopharmacological and psychosocial rehabilitation in oncological patients further stabilizes their quality of life. The recommendations extracted in this study are expected to further improve the successful treatment and rehabilitation of patients with maxillofacial malignancies.

\section{Acknowledgement}

This work is supported, in part, by the non-profit organization Epidemiological \& Clinical Research Information Network (ECRIN).

\section{References}

1) Kaveh K., Inrahim R., Bakar M.Z.A., Ibrahim T.A. Bone grafting and bone graft substitutes. J. Anim. Vet. Edv. 2010; 9: 1055-1067.

2) Kochurova E., Nikolenko V. Immunobiological indicators in oral cavity at the ages of dental rehabilitation of patients with oncology disease. Ros. Stomatol. Jurn. 2014; N 4.

3) Reshetov I., Sergeeva N.S., Polakov A.P. et al. Clinical observation of the reconstruction of the bone defect of maxillofacial area with complex individual composite endoprosthesis. Oncologiya. Jurn. After P. A. Hertsen. 2014; 3: 51-55.

4) Svetitskiy P.V., Zabalueva L.M. Complex prosthesis of the patients with tumors of upper and lower jaws. Swords Greg A., Noubl Eron M. Craniofacial implant / Claim for invention N 2008152069. Bull. N 20 of 20.07.2010.

5) Chuchkov V.M., Kulakov A.A., Matyakin E.G. Medical rehabilitation of the oncological patients with defects of upper jaw. Stomatology. 2009; 2: 50-53.

6) Ubaydullaev K.A., Maeda H., Gafforov A.S., Rizaev J.A., GafurAkhunov M-A. Benefit of rehabilitation for patients with postoperative defects due to maxillofacial tumors. Ann. Cancer Res. Ther. 2019; 27: 19-21.

7) Murashov M.A. Prosthesis after resection of the jaws. The 6th international Scientific-practical conference on reconstructive maxillofacial surgery. Krasnogorsk. 2014.

8) Chissov V.I., Panchenko V.V., Reshetov I.V. et al. Planning in the reconstructive oncosurgery of the maxillofacial area. Improvement of the medical aid in oncological diseases, including actual problems of children's hematology and oncology. National Oncological Program: Sci.- pract.conf. with int.part. 2009; T.2.-C: 193.

9) Oral heals surveys. Basic methods. Geneva: WHO. 2007; 712. 